\title{
Coulomb dominated cavities in bilayer graphene
}

\author{
Marius Eich, ${ }^{1, *}$ Riccardo Pisoni, ${ }^{1}$ Chuyao Tong, ${ }^{1}$ Rebekka Garreis,,${ }^{1}$ Peter Rickhaus $\odot,{ }^{1}$ Kenji Watanabe $\odot,{ }^{2}$ \\ Takashi Taniguchi, ${ }^{2}$ Thomas Ihn ${ }^{\circ},{ }^{1}$ Klaus Ensslin ${ }^{\circ},{ }^{1, \dagger}$ and Annika Kurzmann ${ }^{1}{ }^{1}$ \\ ${ }^{1}$ Solid State Physics Laboratory, ETH Zurich, 8093 Zurich, Switzerland \\ ${ }^{2}$ Advanced Materials Laboratory, NIMS, 1-1 Namiki, Tsukuba 305-0044, Japan
}

(Received 30 January 2020; revised manuscript received 20 April 2020; accepted 22 April 2020; published 12 May 2020)

\begin{abstract}
Electrostatic confinement in bilayer graphene van der Waals heterostructures provides a versatile platform for studying electronic transport in bilayer graphene nanostructures. We study bilayer graphene cavities which we interpret in terms of quantum dots that are strongly coupled to the leads. We investigate how the transport signatures evolve when changing the size of the cavity for both electron and hole occupation. In addition, we analyze the interplay of single and double quantum dot physics in the regime where transport through the quantum dot system is almost pinched off.
\end{abstract}

DOI: 10.1103/PhysRevResearch.2.022038

\section{INTRODUCTION}

Advances in the fabrication of electrostatically defined nanostructures in van der Waals heterostructures [1,2] have enabled the fabrication of quantum point contacts [3] and quantum dots (QDs) [4-6] in bilayer graphene. These devices rely on opening a band gap by applying a strong perpendicular electric field to the bilayer graphene flake [7-9]. Electronic transport in the dual-gated regions of the bilayer graphene device can be strongly suppressed by tuning the Fermi energy into the band gap $[10,11]$. A key ingredient is the utilization of a graphite back gate, which improves the achieved pinch-off resistances [3,12].

Previous publications have reported on single charge carrier occupation [4], charge detection [13], and the excited state spectra of bilayer graphene QDs [14]. For these experiments, the tunnel barriers defining the QDs were tuned into a regime where the width of the Coulomb resonances was dominated by thermal broadening. Here, we report on a single QD in the electron or hole regime which is strongly coupled to the leads such that the tunnel coupling $\Gamma \approx 500 \mu \mathrm{eV}$ exceeds the single particle level spacing and $k_{\mathrm{B}} T$ by far. The Coulomb charging energy, however, remains the dominant energy scale. We show that we can sequentially fill several hundreds of electrons into the QD and discuss how the formation of a double dot leads to a complex beating pattern in the Coulomb blockade resonances. Throughout the manuscript we make a distinction to Fabry- Pérot resonances [15], which are typically measured with open barriers (transmission of the barriers around 50\%)

\footnotetext{
*meich@phys.ethz.ch

†ensslin@phys.ethz.ch
}

Published by the American Physical Society under the terms of the Creative Commons Attribution 4.0 International license. Further distribution of this work must maintain attribution to the author(s) and the published article's title, journal citation, and DOI. but where the electron number in the interferometer is not quantized. Our results describe the open-barrier regime of bilayer graphene QDs.

\section{RESULTS AND DISCUSSION}

We investigated the bilayer graphene van der Waals heterostructure shown in the inset of Fig. 1(a). The heterostructure consists of a roughly $1 \mu \mathrm{m}$ wide, mechanically exfoliated bilayer graphene flake encapsulated in hexagonal boron nitride and features a graphite back gate. The fabrication is described in Refs. [3] and [4]. Metallic top gates are patterned on top of the heterostructure using electron beam lithography and are used to define nanostructures electrostatically [3-5]. The split gate structure [yellow in Fig. 1(a)] is used to confine charge carriers to the roughly $130 \mathrm{~nm}$ wide channel between the two split gates. This is achieved by applying opposite voltages to the split gates and the graphite back gate, which creates a strong displacement field and opens a band gap in the bilayer graphene band structure [7-9]. The chemical potential in the bilayer graphene region underneath the split gates is then tuned into the band gap such that these regions are depleted of charge carriers $[3,4]$. Note that the electronic width of the created channel will be less than $130 \mathrm{~nm}$ because of the finite thickness of the dielectric layers. The finger gates [numbered 1 through 6 in Fig. 1(a)] sitting on top of the split gates but separated from them by a layer of $\mathrm{Al}_{2} \mathrm{O}_{3}$ are then used to locally tune the chemical potential along the channel, to deplete the channel locally, or even to invert the charge carrier polarity [5]. The finger gates are roughly $40 \mathrm{~nm}$ wide and separated by roughly $90 \mathrm{~nm}$. The complete sample (shown in the Supplemental Material) consists of two devices. One device (device 2) is also used in Ref. [4], but here we study the transport with open tunneling barriers on device 1 [16].

The device is measured in a two-terminal setup using a low bias excitation. In an initial cooldown, the device is measured at a temperature of $T=1.7 \mathrm{~K}$. The graphite back gate voltage is set to $V_{\mathrm{BG}}=5.55 \mathrm{~V}$ such that the bilayer graphene 


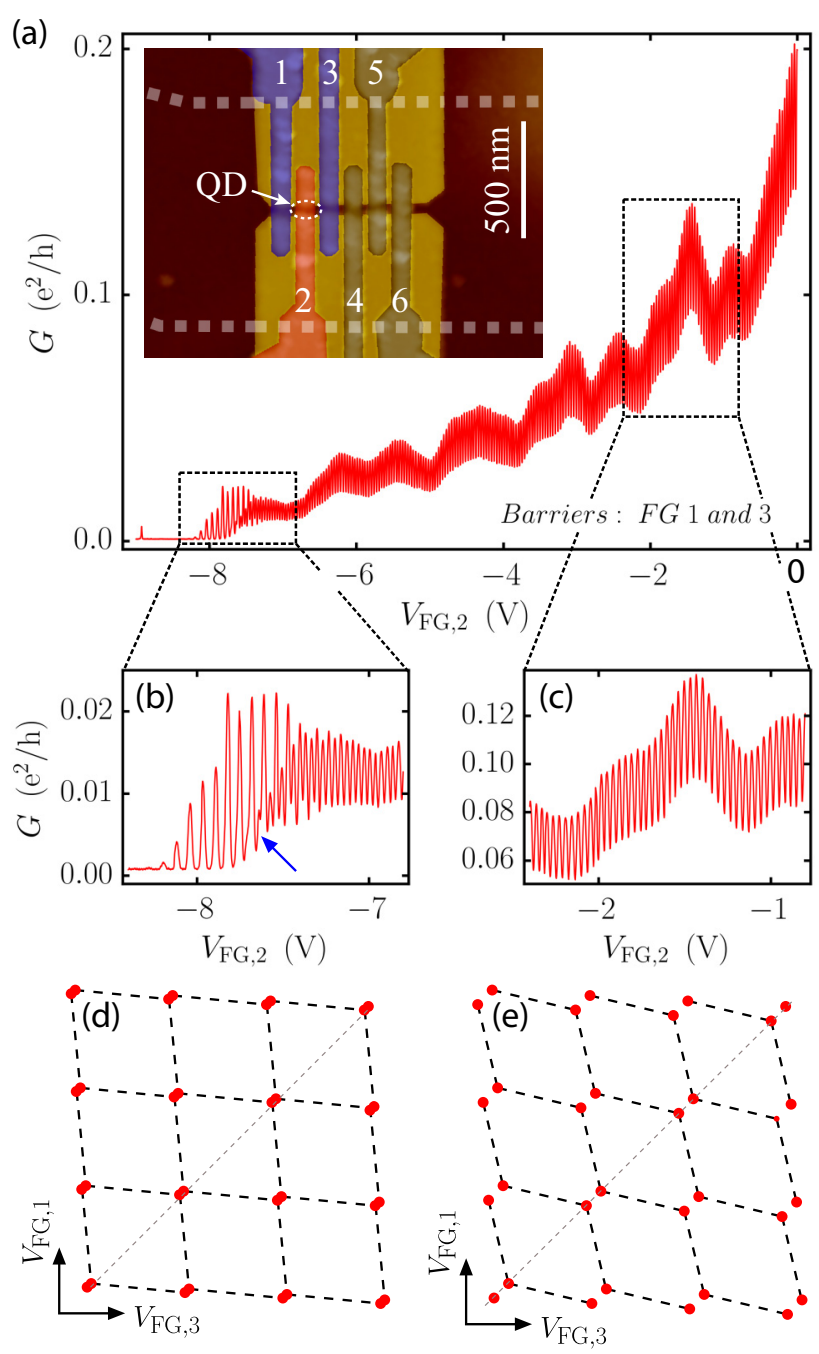

FIG. 1. (a) Conductance $G$ measured as a function of the voltage $V_{\mathrm{FG}, 2}$ applied to finger gate 2 (red in inset), while finger gates 1 and 3 define the tunnel barriers (blue in inset) of the $\mathrm{QD}\left(V_{\mathrm{BG}}=5.55 \mathrm{~V}\right.$, $V_{\mathrm{SG}}=-4.57 \mathrm{~V}, V_{\mathrm{SD}}^{\mathrm{AC}}=0.1 \mathrm{mV}$, and $\left.T=1.5 \mathrm{~K}\right)$. Inset: False color scanning force micrograph of the device. The bilayer graphene flake is outlined by white dashed lines and the split gates defining the channel are shown in yellow, while the finger gates are labeled with numbers 1 through 6 . Selected segments of the current trace are shown in (b) close to pinch-off of the channel and in (c) for a regime where the electron density underneath finger gate 2 is only slightly reduced compared to the overall electron density in the bilayer graphene system. Schematic double QD charge stability diagrams are shown in (d) for weak interdot coupling and (e) for stronger interdot coupling.

system is electron doped (charge neutrality is reached at $V_{\mathrm{BG}}=-0.34 \mathrm{~V}$ in regions not covered by any top gates). The induced electron density in the channel, extracted from a Landau fan measurement, is roughly $n\left(V_{\mathrm{BG}}=5.55 \mathrm{~V}\right)=$ $3.5 \times 10^{12} \mathrm{~cm}^{-2}$. The split gates are biased at $V_{\mathrm{SG}}=-4.57 \mathrm{~V}$ to deplete the bilayer graphene regions underneath the split gate and force electrons to flow through the channel. For the measurement presented in Fig. 1, finger gates 1 and 3 [blue in inset of Fig. 1(a)] are biased at $V_{\mathrm{FG} 1,3}=-7.5 \mathrm{~V}$ close to their respective pinch-off voltage, such that they form two open tunnel barriers along the channel. This creates a roughly $200 \mathrm{~nm}$ long $n$-type electronic cavity inside the channel. Varying the voltage applied to finger gate 2 [red in inset of Fig. 1(a)] tunes the chemical potential inside this cavity such that individual electrons can be added or removed from the cavity. Using this configuration, we observe periodic conductance oscillations (see Fig. 1) as a function of the finger gate voltage $V_{\mathrm{FG}, 2}$. Close to $V_{\mathrm{FG}, 2}=0 \mathrm{~V}$, where the electronic density underneath finger gate 2 is similar to the electronic density in the rest of the cavity, we observe sinusoidal oscillations on top of a conductance background [see Fig. 1(c)]. The amplitude of these oscillations is roughly $0.01 \mathrm{e}^{2} / \mathrm{h}$ and the frequency is $28.76 / \mathrm{V}$.

We interpret the observed conductance oscillations in terms of Coulomb resonances of a large QD. This agrees with the observation that the period of the oscillations is directly related to the addition energy of the QD $[17,18]$. If the tunnel barriers below finger gates 1 and 3 are closed, we observe typical Coulomb diamonds with an addition energy of roughly $1 \mathrm{meV}$ [5] where transport through the QD occurs via a single energy level [19].

The conductance trace in Fig. 1(a) contains roughly 230 oscillations between $V_{\mathrm{FG}, 2}=0 \mathrm{~V}$ and $V_{\mathrm{FG}, 2} \approx-7.5 \mathrm{~V}$, where the bilayer graphene region underneath finger gate 2 is depleted. This implies that roughly 230 electrons are pushed out of the QD. Assuming that the QD has a homogeneous electron density of $n=3.5 \times 10^{12} \mathrm{~cm}^{-2}$ at $V_{\mathrm{FG}, 2}=0 \mathrm{~V}$, the number of oscillations $n_{e}$ can be used to determine which fraction of the QD's area $A$ is directly influenced by finger gate 2, by using

$$
n \cdot A \approx n_{e} .
$$

The resulting area of roughly $6600 \mathrm{~nm}^{2}$ leads to an area with $60 \mathrm{~nm} \times 110 \mathrm{~nm}$, which agrees well with the lithographic design of the device (due to the finite thickness of the dielectric layers on top of the bilayer graphene the electronic channel is slightly smaller than the gap between the split gates and the finger gates influence a slightly larger part of the channel than lithographically designed). To determine if the addition energy of the QD is dominated by the lateral confinement or the Coulomb interaction we estimate the two energy scales. With $A$ being the area of the $\mathrm{QD}$, the characteristic confinement energy $\Delta$ is given by $\Delta=\left(\pi \hbar^{2}\right) /\left(4 m^{*} A\right) \approx 100 \mu \mathrm{eV}$, while the characteristic Coulomb energy scale $E_{C}$ is given by $E_{C}=\left(e^{2}\right) /\left(8 \epsilon \epsilon_{0} \sqrt{A / \pi}\right) \approx 5 \mathrm{meV}$ [18]. In contrast to the QDs reported on in Ref. [4], the addition energy of the QDs studied here is clearly dominated by the Coulomb energy. As a consequence, the oscillation frequency in Fig. 1(a) is independent of the QD occupation.

Figure 1(b) shows a closeup of the conductance trace around the pinch-off voltage at $V_{\mathrm{FG}, 2} \approx 7.5 \mathrm{~V}$, where the bilayer graphene region below finger gate 2 is almost depleted. In this regime we observe a beating pattern with an increase (roughly doubling) of the oscillation frequency as the finger gate voltage $V_{\mathrm{FG}, 2}$ is increased. The large QD formed between finger gates 1 and 3 can be cut into two parts by partially depleting the electron gas below finger gate 2, which occurs for $V_{\mathrm{FG}, 2} \approx-8 \mathrm{~V}$ (shown in [5] and [20]). In this situation, two QDs are formed - one between finger gates 1 and 2 and a second one between finger gates 2 and 3 . By increasing $V_{\mathrm{FG}, 2}$, 

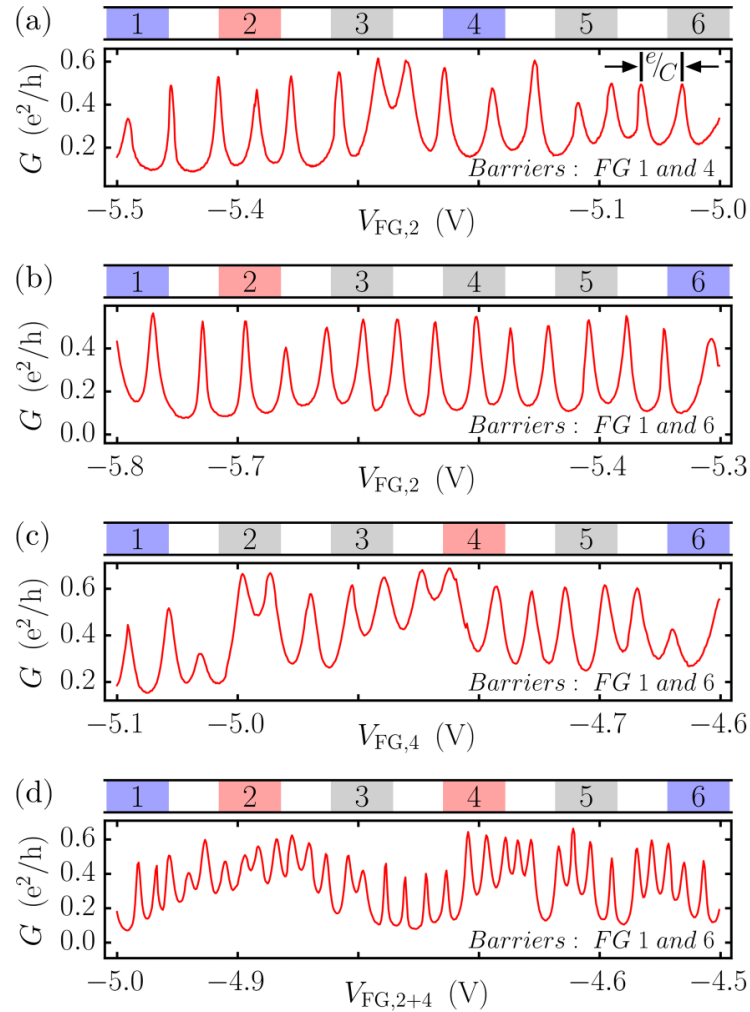

FIG. 2. Conductance $G$ measured with an applied bias voltage of $V_{\mathrm{SD}}^{\mathrm{DC}}=10 \mu \mathrm{V}\left(V_{\mathrm{BG}}=3 \mathrm{~V}, V_{\mathrm{SG}}=-2.9 \mathrm{~V}\right.$, and $\left.T=100 \mathrm{mK}\right)$. The schematic on top of each subplot indicates which finger gate is driven (shown in red) and which finger gates define the tunnel barriers (shown in blue). Note that the frequency of the conductance oscillations in (d) is doubled compared to (a)-(c).

the tunnel coupling between these two QDs becomes stronger until they form a single QD where the multiparticle wave function extends over the whole electronic cavity between finger gates 1 and 3. The double QD regime is characterized by a hexagonal charge stability diagram [schematically shown in Figs. 1(d) and 1(e)] when sweeping the gate voltages applied to finger gates 1 and $3[5,20]$. Sweeping $V_{\mathrm{FG}, 2}$ then corresponds to a diagonal cut through the charge stability diagram, where we cut (gray dashed lines) through the triple points [red dots in Figs. 1(d) and 1(e); see also [16]] where charge transport through the double QD system is possible. For increasing $V_{\mathrm{FG}, 2}$, the coupling between the two halves of the double QD is increased such that the splitting between the triple points in the charge stability diagram increases, leading to a transition from Figs. 1(d) to 1(e). In the experiment, this is seen as a shoulder developing on the low $V_{\mathrm{FG}, 2}$ side of the Coulomb resonances, which is indicated by the blue arrow in Fig. 1(b). By increasing $V_{\mathrm{FG}, 2}$ further, the triple points split further apart leading to two distinct conductance resonances and the frequency of the oscillations is doubled in this device $[5,21]$.

Since thermal broadening strongly influences the oscillation pattern, the device was cooled down in a dilution refrigerator to $T=100 \mathrm{mK}$. Figure 2 presents a series of measurements where we change the finger gates forming the tunnel barriers and also the finger gate tuning the number of electrons inside the resulting QD (referred to as the plunger gate). First, we change the length of the QD compared to the measurement presented in Fig. 1 by shifting one tunnel barrier from finger gate 3 to finger gate 4 in Fig. 2(a) and finger gate 6 in Fig. 2(b). Compared to the QD studied in Fig. 1 the resulting QDs are roughly 1.6 and 3 times larger, respectively. Sweeping finger gate voltage $V_{\mathrm{FG}, 2}$ again leads to a series of conductance oscillations. Compared to the sinusoidal conductance oscillations in Fig. 1(c) the oscillations in Fig. 2 appear as overlapping Lorentzian peaks, which we attribute to the reduced thermal broadening of the Coulomb resonances. The oscillation frequencies are $30.61 / \mathrm{V}$ for the roughly $320 \mathrm{~nm}$ long QD [Fig. 2(a)] and 31.09/V for the roughly $600 \mathrm{~nm}$ long QD [Fig. 2(b)]. Therefore, the oscillation frequency is almost independent of the length of the QD. The period of the oscillations is given by $e / C$ [see Fig. 2(a)], where $C$ is the capacitance between the QD and the plunger gate. This capacitance is determined by the lithographic design of the sample - mainly by the width of the electronic channel, the width of the finger gate, and the thickness of the dielectric layers separating the two. Since the capacitance does not depend on the size of the QD, the period of the conductance oscillations is also independent of the QD size.

For the measurement presented in Fig. 2(c) we use finger gate 4 as the plunger gate instead of finger gate 2 , while all the other voltages are kept constant. Since the lithographic design for all finger gates is the same, we expect the capacitances between individual finger gates and the electronic channel to be roughly the same. As expected, the conductance oscillations in Fig. 2(c) closely resemble the ones in Fig. 2(b). The frequency of the oscillations in Fig. 2(c) is 33.46/V. We attribute the slight variation in the oscillation frequency to a slight variation in the plunger gate capacitance when switching from finger gate 2 to 4 , which can result from microscopic variations in the lithographic gate structure. Finally, in Fig. 2(d) we drive finger gates 2 and 4 simultaneously, again while keeping all the other voltages the same. When driving both finger gates simultaneously, the total capacitance is the sum of the two individual capacitances. The observed conductance oscillations now have a higher frequency of $65.35 / \mathrm{V}$, which agrees well with the sum of the frequencies extracted from Figs. 2(b) and 2(c).

For Fabry-Pérot oscillations in a cavity one would expect the periodicity of the oscillations to scale with the gated length of the cavity. If the cavity were viewed as a twodimensional (2D) system similar to the experiments presented in Ref. [15], the distance between conductance minima in density and therefore in gate voltage is expected to change with the number of modes. The data presented in Fig. 1(a) shows that there is a constant number of about 29 minima per volt in the gate voltage range between $-8 \mathrm{~V}$ and $0 \mathrm{~V}$ covering about 10 to 240 modes. This clearly shows that our data cannot be explained by a 2D noninteracting Fabry-Pérot cavity. How about Fabry-Pérot oscillations in a one-dimensional (1D) cavity, similar to results observed in carbon nanotubes [22]? The area underneath the finger gate is roughly $60 \mathrm{~nm} \times 110 \mathrm{~nm}$ large. The data in Fig. 1(a) shows about 220 oscillations. So it is highly unlikely that only one transverse mode would exist under the tuning gate for the entire gate voltage range. If one nevertheless assumes a $1 \mathrm{D}$ cavity and approximates the $1 \mathrm{D}$ 


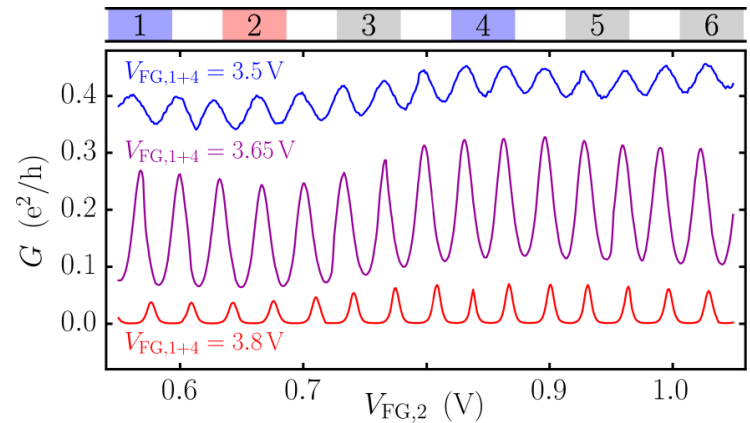

FIG. 3. Conductance $G$ measured with an applied bias voltage of $V_{\mathrm{SD}}^{\mathrm{DC}}=10 \mu \mathrm{V}\left(V_{\mathrm{BG}}=-5 \mathrm{~V}\right.$ and $\left.V_{\mathrm{SG}}=2.464 \mathrm{~V}\right)$. Note that the negative back gate voltage induces an overall hole doping in the bilayer graphene system. (a) The finger gate voltage $V_{\mathrm{FG}, 2}$ controls the hole occupation of the $\mathrm{QD}$, while $V_{\mathrm{FG}, 1+4}$ applied to finger gates 1 and 4 simultaneously controls the tunnel coupling of the QD to the leads at $T=500 \mathrm{mK}$.

density by the square root of the 2D density, one would expect a period of roughly $1 \mathrm{~V}$ for the oscillations, which clearly does not match the experimental result. We conclude that Coulomb interaction between individual electrons inside the cavity is the dominant mechanism and that a noninteracting Fabry-Pérot analysis cannot be applied.

So far, we have applied a positive graphite back gate voltage $V_{\mathrm{BG}}>0$ resulting in an overall electron doping of the bilayer graphene system. Due to the ambipolar nature of bilayer graphene, we can repeat all the experiments with inverted gate voltages [4]. Applying a negative voltage $V_{\mathrm{BG}}<$ 0 will induce an overall hole doping and by applying corresponding voltages to the split gates and finger gates, we can form a $p$-type QD $[4,5]$. This situation is presented in Fig. 3, where finger gates 1 and 4 define the tunnel barriers of the QD. Again, we use finger gate 2 as the plunger gate. In addition, we can vary the voltage applied to finger gates 1 and 4 to change the tunnel coupling of the QD to the leads [23]. In Fig. 3 we show cuts for varying voltage $V_{\mathrm{FG}, 1+4}$ applied to finger gates 1 and 4. Consequently, we observe that the overall conductance of the system is lowered, as the voltage applied to finger gates 1 and 4 is increased. As $V_{\mathrm{FG}, 1+4}$ is increased, the conductance oscillations evolve from sinusoidal conductance oscillations (blue, $V_{\mathrm{FG}, 1+4}=3.5 \mathrm{~V}$ ), where the tunnel coupling to the leads is large, to temperature-broadened Coulomb resonances (red, $V_{\mathrm{FG}, 1+4}=3.8 \mathrm{~V}$ ), where the tunnel coupling of the QD to the leads is small.

The visibility of the conductance oscillations is largest for an intermediate coupling (purple, $V_{\mathrm{FG}, 1+4}=3.65 \mathrm{~V}$ ). Without tunnel barriers being defined underneath finger gates 1 and 4 , the conductance is largely independent of $V_{\mathrm{FG}, 2}$ over small ranges in $V_{\mathrm{FG}, 2}$ close to $V_{\mathrm{FG}, 2}=0 \mathrm{~V}$ [4]. When forming the tunnel barriers, the conductance traces start to exhibit oscillations with a small amplitude (blue trace in Fig. 3). The visibility of these oscillations initially increases as $V_{\mathrm{FG}, 1+4}$ is increased (purple trace in Fig. 3). As the tunnel coupling of the QD to the leads is decreased further by increasing $V_{\mathrm{FG}, 1+4}$, the conductance resonances should become infinitely sharp in the zero-temperature limit, while the peak amplitude

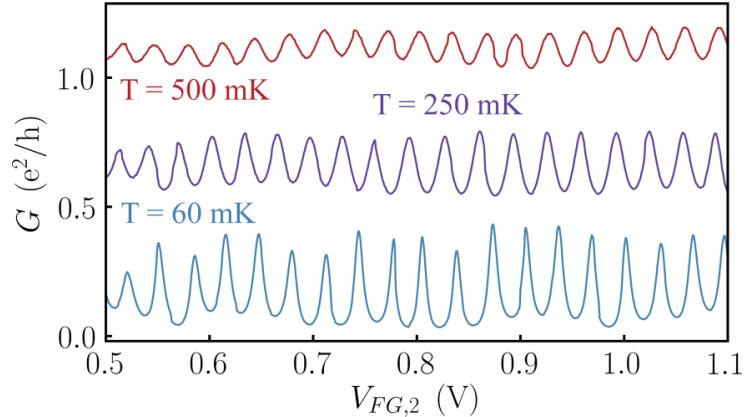

FIG. 4. Conductance $G$ measured with an applied bias voltage of $V_{\mathrm{SD}}^{\mathrm{DC}}=10 \mu \mathrm{V}\left(V_{\mathrm{BG}}=-5 \mathrm{~V}\right.$ and $\left.V_{\mathrm{SG}}=2.464 \mathrm{~V}\right)$. The finger gate voltage $V_{\mathrm{FG}, 2}$ controls the hole occupation of the $\mathrm{QD}$, while $V_{\mathrm{FG}, 1+4}$ applied to finger gates 1 and 4 forms the tunnel barrier between the QD and the leads at different temperatures. Decreasing the temperature for $V_{\mathrm{FG}, 2}=3.6 \mathrm{~V}$ shows the thermal broadening of the Coulomb resonances.

stays constant. However, finite temperature leads to thermal broadening of these resonances, keeping the area underneath the Coulomb resonances constant (see Fig. 4). Therefore, the peak amplitude is reduced as the tunnel coupling to the leads is reduced further.

Figure 4 shows the temperature dependence of the conductance oscillation for base temperatures between 60 and $500 \mathrm{mK}$ using the finger gates as shown in Fig. 3. A broadening of the resonances and a decrease of the conductance amplitude is observed, when the temperature is increased, as expected for a temperature broadening of Coulomb resonances.

\section{CONCLUSION}

In conclusion, we form QDs which are strongly coupled to the leads by exploiting the versatility of electrostatically defined nanostructures in bilayer graphene. With a set of finger gates on top of a narrow conducting channel, we can vary the length of the QD. Each finger gate can form a tunnel barrier or serve as the plunger gate to the resulting QD controlling the number of charge carriers in the QD. The frequency of the observed Coulomb resonances is given by the capacitance between the plunger gate and the QD and it is therefore determined by the lithographic design of the sample. When changing the plunger gate voltage, all the charge carriers below the plunger gate can be removed from the QD. This allows us to determine which fraction of the QD's area is influenced by the plunger gate. In the regime where this area is almost depleted, double QD transport signatures start to emerge as an additional tunnel barrier is formed underneath the plunger gate. As a consequence, an increase (roughly doubling) of the conductance oscillation frequency is observed. All presented experiments can be carried out in an electron or hole-dominated regime due to the ambipolar nature of bilayer graphene. The tunnel coupling of the QD to the leads can be easily tuned by changing the voltage applied to the finger gates forming the tunnel barriers and we observe 
that the visibility of the conductance oscillations is largest for an intermediate tunnel coupling to the leads.

The open barrier regime presented in this paper is a key requirement for observing the Kondo effect in graphene QDs which requires further experiments. The large tunability of electrostatically defined QD systems in bilayer graphene together with the implementation of charge detection and possibly resonator readout schemes opens up a wide field of graphene-based quantum information processing which promises long coherence times. In addition, improved plunger gate designs will enable studying the interplay of the valley degree of freedom and spin-blockade physics in bilayer graphene double QDs.

\section{ACKNOWLEDGMENTS}

We thank P. Märki and T. Bähler as well as the FIRST staff for their technical support. We also acknowledge financial support from the European Graphene Flagship, the Swiss National Science Foundation via NCCR Quantum Science and Technology, the EU Spin-Nano RTN network, ETH Zurich via the ETH fellowship program, and the European Union's Horizon 2020 research and innovation programme under the Marie Skłodowska-Curie Grant Agreement No. 766025. Growth of hexagonal boron nitride crystals was supported by the Elemental Strategy Initiative conducted by the MEXT, Japan and JSPS KAKENHI Grant No. JP15K21722.
[1] L. Wang, I. Meric, P. Y. Huang, Q. Gao, Y. Gao, H. Tran, T. Taniguchi, K. Watanabe, L. M. Campos, D. A. Muller, J. Guo, P. Kim, J. Hone, K. L. Shepard, and C. R. Dean, Science 342, 614 (2013)

[2] P. J. Zomer, M. H. D. Guimaräes, J. C. Brant, N. Tombros, and B. J. van Wees, Appl. Phys. Lett. 105, 013101 (2014).

[3] H. Overweg, H. Eggimann, X. Chen, S. Slizovskiy, M. Eich, R. Pisoni, Y. Lee, P. Rickhaus, K. Watanabe, T. Taniguchi, V. Fal'ko, T. Ihn, and K. Ensslin, Nano Lett. 18, 553 (2018).

[4] M. Eich, F. Herman, R. Pisoni, H. Overweg, A. Kurzmann, Y. Lee, P. Rickhaus, K. Watanabe, T. Taniguchi, M. Sigrist, T. Ihn, and K. Ensslin, Phys. Rev. X 8, 031023 (2018).

[5] M. Eich, R. Pisoni, A. Pally, H. Overweg, A. Kurzmann, Y. Lee, P. Rickhaus, K. Watanabe, T. Taniguchi, K. Ensslin, and T. Ihn, Nano Lett. 18, 5042 (2018).

[6] L. Banszerus, B. Frohn, A. Epping, D. Neumaier, K. Watanabe, T. Taniguchi, and C. Stampfer, Nano Lett. 18, 4785 (2018).

[7] E. McCann, Phys. Rev. B 74, 161403(R) (2006).

[8] T. Ohta, A. Bostwick, T. Seyller, K. Horn, and E. Rotenberg, Science 313, 951 (2006).

[9] J. B. Oostinga, H. B. Heersche, X. Liu, A. F. Morpurgo, and L. M. K. Vandersypen, Nat. Mater. 7, 151 (2008).

[10] A. S. M. Goossens, S. C. M. Driessen, T. A. Baart, K. Watanabe, T. Taniguchi, and L. M. K. Vandersypen, Nano Lett. 12, 4656 (2012).

[11] M. T. Allen, J. Martin, and A. Yacoby, Nat. Commun. 3, 934 (2012).

[12] A. A. Zibrov, C. Kometter, H. Zhou, E. M. Spanton, T. Taniguchi, K. Watanabe, M. P. Zaletel, and A. F. Young, Nature (London) 549, 360 (2017).
[13] A. Kurzmann, H. Overweg, M. Eich, A. Pally, P. Rickhaus, R. Pisoni, Y. Lee, K. Watanabe, T. Taniguchi, T. Ihn, and K. Ensslin, Nano Lett. 19, 5216 (2019).

[14] A. Kurzmann, M. Eich, H. Overweg, M. Mangold, F. Herman, P. Rickhaus, R. Pisoni, Y. Lee, R. Garreis, C. Tong, K. Watanabe, T. Taniguchi, K. Ensslin, and T. Ihn, Phys. Rev. Lett. 123, 026803 (2019).

[15] A. Varlet, M.-H. Liu, V. Krueckl, D. Bischoff, P. Simonet, K. Watanabe, T. Taniguchi, K. Richter, K. Ensslin, and T. Ihn, Phys. Rev. Lett. 113, 116601 (2014).

[16] See Supplemental Material at http://link.aps.org/supplemental/ 10.1103/PhysRevResearch.2.022038 for sample and device design.

[17] L. P. Kouwenhoven, D. G. Austing, and S. Tarucha, Rep. Prog. Phys. 64, 701 (2001).

[18] T. Ihn, Semiconductor Nanostructures: Quantum States and Electronic Transport (Oxford University Press, Oxford, New York, 2009).

[19] C. W. J. Beenakker, Phys. Rev. B 44, 1646 (1991).

[20] See Supplemental Material at http://link.aps.org/supplemental/ 10.1103/PhysRevResearch.2.022038 for detailed measurements of the formation of a double dot.

[21] M. Eich, Electrostatically defined quantum dots in bilayer graphene, Doctoral thesis, ETH Zurich, 2019.

[22] W. Liang, M. Bockrath, D. Bozovic, J. H. Hafner, M. Tinkham, and H. Park, Nature (London) 411, 665 (2001).

[23] See Supplemental Material at http://link.aps.org/supplemental/ 10.1103/PhysRevResearch.2.022038 for measurements of the n-type dot. 